Revista Tecné, Episteme y Didaxis: TED. Año 2014, Número Extraordinario. ISSN Impreso: 0121-3814, ISSN web: 2323-0126 Memorias, Sexto Congreso Internacional sobre Formación de Profesores de Ciencias. 08 al 10 de octubre de 2014, Bogotá

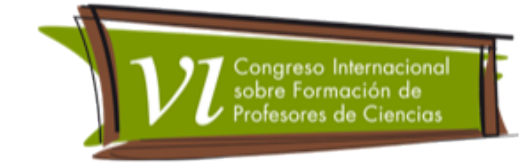

\title{
Intereses E Inquietudes De Los Estudiantes: El Caso De Los Trabajos De Grado De La Especialización En Educación Y Gestión Ambiental Universidad Distrital FJC
}

Salamanca Ana Maritza', Molina Adela², Melo Nadenka³

Categoría 2: Trabajo de investigación

\section{Resumen}

Esta comunicación se inscribe en la Línea de investigación: Enseñanza de las ciencias, contexto y diversidad cultural desarrollada en la Universidad Distrital y se pregunta por el tratamiento dado a los intereses e inquietudes de los niños y niñas dado en los trabajos de grado de la especialización en educación y gestión ambiental de la Universidad Distrital FJC, específicamente en el tema de manejo de residuos sólidos. La metodología se basó en el enfoque Mapeamiento Informacional Bibliográfico (MBI) y se analizaron 80 RAE's (Molina, 2005)

Los resultados muestran una mayor tendencia a la formación de valores y el trabajo con la comunidad como respuesta a los problemas ambientales del entorno, y los intereses e inquietudes de los niños y niñas no se tienen en cuenta aún en los asociados al tema del manejo de residuos sólidos.

\section{Palabras Clave}

Intereses, inquietudes, revisión bibliográfica, residuos sólidos.

\section{Marco Teórico}

En Bogotá el plan de desarrollo económico, social, ambiental y de obras publicas 2012-2016 de Bogotá humana, propone el desarrollo del programa basura cero, que específicamente hace mención a la cultura de reducción de basuras y separación en la fuente, la cual se orienta hacia la formación y sensibilización de los ciudadanos, haciendo uso de campañas masivas sobre el beneficio del

\footnotetext{
${ }^{1}$ Universidad Distrital Francisco José de Caldas salamaritza@gmail.com

${ }^{2}$ Universidad Distrital Francisco José de Caldas mara.gracia@gmail.com

${ }^{3}$ Universidad Distrital Francisco José de Caldas nadenkamelo@gmail.com
} 
Revista Tecné, Episteme y Didaxis: TED. Año 2014, Número Extraordinario. ISSN Impreso: 0121-3814, ISSN web: 2323-0126

Memorias, Sexto Congreso Internacional sobre Formación de Profesores de Ciencias. 08 al 10 de octubre de 2014, Bogotá

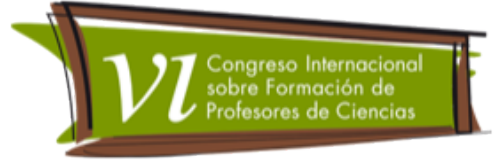

reciclaje, modo de separar en la fuente, disposición diferenciada de residuos sólidos y de igual manera hace referencia a las intervenciones diferenciadas: colegios, universidades, hogares, conjuntos residenciales, negocios, locales comerciales e industrias (Bogotá, 2012). Esta política se corresponde con todas las alertas dadas por organismos nacionales e internacionales

Atendiendo a enfoques socio-científicos y culturales en la enseñanza de las ciencias se requiere un modelo didáctico mediador que permita actuaciones concretas, críticas y coherentes con los resultados arrojados por la investigación científica y didáctica específicamente en el tema de manejo de residuos sólidos por parte de los estudiantes de primer ciclo de un colegio de Bogotá. Lo anterior, con el fin de no caer en el activismo, en la realización de actividades sin entender por qué se hacen, de manera que supere el énfasis dado a la descripción del problema y no a su comprensión, causas y capacitación para la acción sobre el mismo. Los estudiantes se implican realmente si el problema tiene sentido para ellos, es decir si están motivados, si conecta con sus intereses y preocupaciones, con su vida cotidiana, si se tiene claro que se quiere conseguir y cuál es la finalidad del trabajo a realizar.(García, 2004). De esta manera resulta importante conocer las ideas de los otros, su génesis, conocer los intereses económicos y políticos que hay detrás de determinadas verdades.

Al respecto Sanmartí expresa que aquello que se trabaje en clase debe tener sentido para el estudiante, se debe conectar con sus conocimientos previos, con sus intereses, con su lógica, no debe ser la simplificación del saber de los expertos, sino que debe ser una reelaboración de nuevo conocimiento construido en el aula con el propósito de promover el aprendizaje de los estudiantes (Sanmarti, 2010)

Rivarosa plantea que la educación ambiental no se debe impartir en lecciones impuestas por el docente sino por medio de la resolución de problemas ambientales que surgen de la experiencia diaria de los niños y se deben conectar con los interese e inquietudes de los niños, de manera que cobren sentido para ellos para que los puedan aplicar a su vida cotidiana movilizando también contenidos culturales. (Rivarosa, 2006)

Según Geertz la cultura es un conjunto de significados entretejidos en un contexto específico que le permiten a un individuo interpretar el mundo. Esta perspectiva pone su atención en el niño como sujeto que aprende y en su contexto cultural 
Revista Tecné, Episteme y Didaxis: TED. Año 2014, Número Extraordinario. ISSN Impreso: 0121-3814, ISSN web: 2323-0126

Memorias, Sexto Congreso Internacional sobre Formación de Profesores de Ciencias. 08 al 10 de octubre de 2014, Bogotá

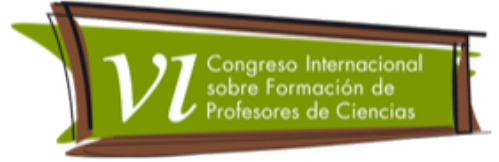

desde el punto de vista de las creencias y perspectivas sobre el mundo ya que enseñanza de las ciencias se debe dar en un contexto de intercambio cultural (Macias, 2008)

Como lo menciona Schön, el conocimiento teórico o académico forma parte de la reflexión, cuando se integra de manera significativa en la mente del niño de manera que haga parte de un pensamiento práctico. (Schön, 1992).

Las ATAs (Actividades Totalidad Abiertas) son una alternativa didáctica que permite recuperar lo cotidiano como objeto de estudio, problematizar, cuestionar las reducciones empobrecedoras del objeto, enriquecer los fenómenos, articular los conocimientos que se construyen con los conocimientos que tienen los niños y las niñas, seleccionar los temas a tratar partiendo de los intereses e inquietudes de ellos y su profundidad dependerá de su desarrollo intelectual, (Segura D. M., 2000). Al respecto Molina (Molina, 2007, pág. 91) al tratar las acciones de los niños y de las niñas frente a los problemas ambientales y sus vivencias, plantea que: "Una posible especialización de sus vivencias inmediatas, tal como los mismos niños y niñas proponen, sería pasar a la acción en el contexto escolar, en la cual, se involucran ellos mismos como actores de la propia solución; igualmente en este caso, se mantiene una valoración de lo que es bueno y malo; Puche(2000) da un ejemplo de especialización de la experiencia, en este modo, se refiere a la capacidad de elaborar complejas estrategias de anticipación frente a la conducta de los demás (Puche, R. y otros 2000, pág. 147)

\section{Metodología}

El objetivo fue realizar identificar tendencias en los trabajos de la especialización en educación y gestión ambiental de la Universidad Distrital FJC, específicamente cómo son considerados los intereses e inquietudes de los niños y las niñas en las propuestas educativas

Se revisarán RAE's (Molina, 2005) correspondientes a 80 trabajos de grado de especialización en educación y gestión ambiental de la universidad Distrital FJC, realizados entre los años 2000 y 2002 relacionados con el tema de residuos sólidos.

Así lo que interesa responder es ¿̀ as actividades del aula permiten incorporar los intereses e inquietudes de los estudiantes, específicamente en el tema de manejo de residuos sólidos? 
Revista Tecné, Episteme y Didaxis: TED. Año 2014, Número Extraordinario. ISSN Impreso: 0121-3814, ISSN web: 2323-0126 Memorias, Sexto Congreso Internacional sobre Formación de Profesores de Ciencias. 08 al 10 de octubre de 2014, Bogotá

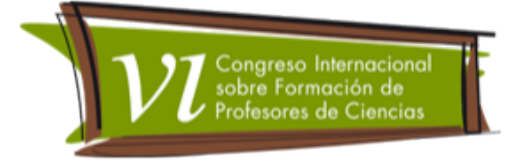

La metodología MBI implica que al mapear un contenido se debe sintetizarlo, lo que requiere una lectura atenta de las informaciones, su comprensión, la identificación de las ideas principales del autor y su registro escrito de modo conciso, coherente y objetivo (André,2009; Cremades, 2011; Molina 2012) citados por (Molina, 2013)

\section{Resultados y análisis}

En cuanto a la selección de las fuentes documentales se utilizaron, palabras clave y una primera lectura de los Resúmenes Analíticos Educativos (Molina, 2005). En el análisis se dará importancia a las temáticas abordadas por los autores, sus tendencias y la relación de éstas con los intereses e inquietudes de los niños y las niñas. La información se organizó en un archivo Excel y se introdujo en una hoja de cálculo (ver Tabla Nº 1)

Tabla N¹. Descripción de ítems para organizar la información.

\begin{tabular}{|c|c|c|c|c|c|c|c|c|c|c|c|c|c|c|c|}
\hline $\begin{array}{c}\text { Tendencia } \\
\text { s } \\
\text { encontrad }\end{array}$ & \multicolumn{4}{|c|}{ Ambiental } & \multicolumn{3}{|c|}{ Educativa } & \multicolumn{4}{|c|}{ Sociocultural } & \multicolumn{4}{|c|}{ Técnica } \\
\hline $\begin{array}{l}\text { Temáticas } \\
\text { abordada } \\
\text { s }\end{array}$ & 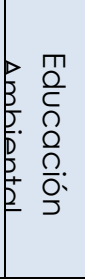 & $\begin{array}{l}\frac{D}{3} \\
\frac{0}{D} \\
\frac{D}{D} \\
\frac{D}{D}\end{array}$ & 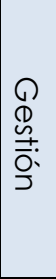 & $\begin{array}{l}0 \\
0 \\
3 \\
\frac{1}{2} \\
\frac{2}{0} \\
0 \\
0\end{array}$ & $\begin{array}{l}\text { 而 } \\
\text { 而 }\end{array}$ & 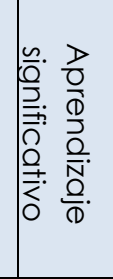 & $\begin{array}{l}0 \\
0 \\
0 \\
0 \\
0 \\
0 \\
0 \\
0 \\
0 .\end{array}$ & 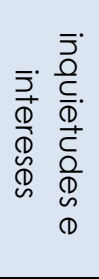 & 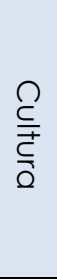 & 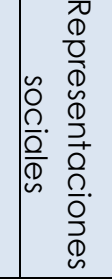 & $\begin{array}{l}\frac{\alpha}{O} \\
\frac{O}{0} \\
\frac{D}{0}\end{array}$ & 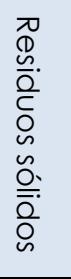 & 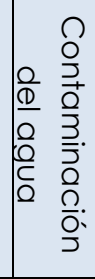 & 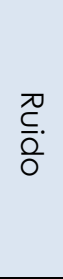 & 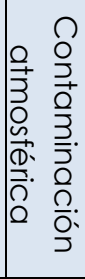 \\
\hline $\begin{array}{c}\mathrm{N}^{\circ} \text { de } \\
\text { trabajos } \\
\text { por } \\
\text { temática y } \\
\text { tendencia }\end{array}$ & 55 & 23 & 7 & 27 & 7 & 11 & 19 & 1 & 5 & 3 & 45 & 17 & 10 & 3 & 2 \\
\hline $\begin{array}{c}\text { Total } \\
\text { trabajos } \\
\text { por } \\
\text { Tendencia }\end{array}$ & & \multicolumn{3}{|c|}{119} & & 30 & & \multicolumn{4}{|c|}{54} & \multicolumn{4}{|c|}{32} \\
\hline
\end{tabular}


Revista Tecné, Episteme y Didaxis: TED. Año 2014, Número Extraordinario. ISSN Impreso: 0121-3814, ISSN web: 2323-0126

Memorias, Sexto Congreso Internacional sobre Formación de Profesores de Ciencias. 08 al 10 de octubre de 2014, Bogotá

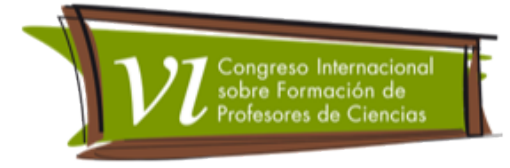

Figura $\mathbf{N}^{\circ} 1$ Relación de tendencias $V_{s} N^{\circ}$ de trabajos.

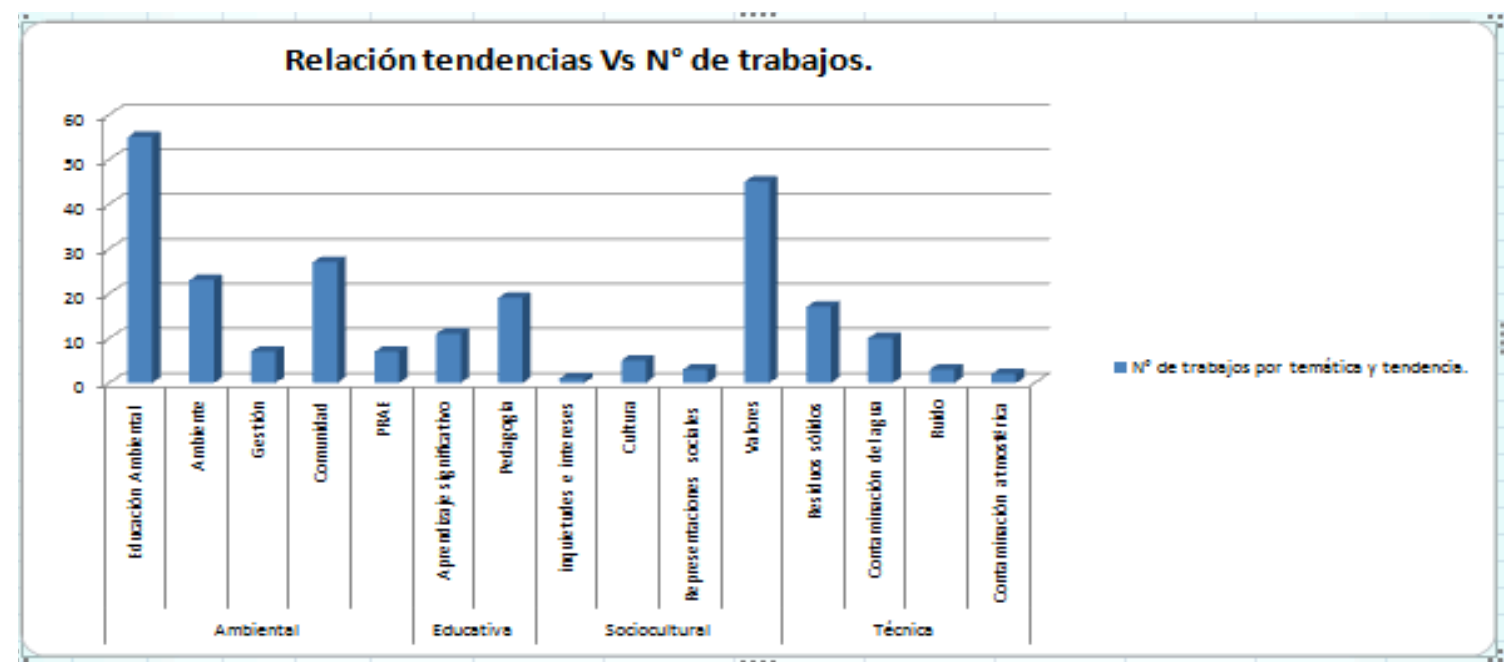

Fuente. Elaborado por el equipo de investigación a partir de (Molina, 2005)

Los resultados muestran una mayor tendencia a la formación de valores y el trabajo con la comunidad como respuesta a los problemas ambientales del entorno, y los intereses e inquietudes de los niños y niñas no se tienen en cuenta aún en los asociados al tema del manejo de residuos sólidos.

Respecto a la tendencia ambiental se dio importancia a la relación entre actores sociales y los ambientes naturales, Intervención en el proceso de planeación de la localidad y uso del recurso público en aspecto ambiental por las juntas de acción comunal ASOJUNTAS; manejo, diseño e implementación de los PRAE"s en los colegios como acción interdisciplinaria a la solución de problemas ambientales de una localidad, generación de valores éticos en la comunidad educativa y evaluación de la función de los PRAE's .

En la tendencia educativa se dio integración de problemas ambientales del entorno y currículo con la generación de espacios de investigación que articulan el saber específico con el componente ecológico; descripción del modelo pedagógico ambiental de comunidades indígenas que transmiten sus saberes y tradición cultural generación tras generación; contraste entre conceptos relativos 
Revista Tecné, Episteme y Didaxis: TED. Año 2014, Número Extraordinario. ISSN Impreso: 0121-3814, ISSN web: 2323-0126

Memorias, Sexto Congreso Internacional sobre Formación de Profesores de Ciencias. 08 al 10 de octubre de 2014, Bogotá

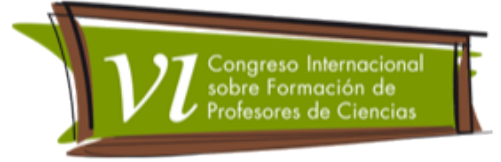

al medio ambiente y los valores en estudiantes de licenciatura en biología en relación al proyecto educativo universitario; la evaluación de los PRAE's como propuestas de transformación educativa que posibilita una nueva forma de relación con la sociedad y con la naturaleza pero que presenta dificultades en cuanto a la falta de asesoría técnica, profesional y financiera; críticas a la política de educación ambiental contenida en la ley general de educación y que se basa en el concepto de desarrollo sostenible que impide un sistema de producción y consumo que respete la naturaleza; propuesta teórico metodológica de la enseñanza de la educación ambiental con miras a mejorar el ambiente de una institución educativa y la calidad de vida de sus participantes, diseño de estrategias educativas interactivas para determinar una problemática ambiental y plantear alternativas de solución para las mismas.

El interés de la tendencia sociocultural es la realización de las acciones orientadas a la toma de conciencia del estado ambiental de las localidades. Así se puede encontrar: herramientas tecnológicas que permiten a los ciudadanos conocer más sobre una localidad como es su ubicación geográfica, historia, costumbres y cultura por medio de software y cartillas verdes, reconocimiento de la flora y fauna de un espacio físico que permita prácticas y ensayos a nivel de subproyectos como semilleros, huerta, lombricultura, el uso de componentes lúdicos, artísticos pedagógicos y éticos que contenga el componente ambiental de manera transversal y que permitan mejorar el desempeño y rendimiento de los estudiantes, aún de aquellos con necesidades Educativas Especiales asociados a las condiciones físicas del entorno escolar o de aquellos con dificultades sociales, culturales y educativas. A nivel del manejo de los residuos sólidos se realizó exploración de las representaciones sociales y gestión en educación ambiental participativa en estudiantes y habitantes, estudio de impacto ambiental generado en la sección de frutas de una plaza de mercado y se utilizó la investigación etnográfica como herramienta que incorpora inquietudes e intereses de los estudiantes lo cual permite un cambio de actitud hacia el entorno.

La tendencia técnica preocupada por determinar la contaminación de las localidades debida al crecimiento y expansión que ha experimentado Bogotá y su relación con el uso irracional de bienes y servicios ambientales. Esto se ve reflejado en la baja calidad de vida debida a los problemas ambientales generados por la cercanía de botaderos, de industrias de extracción de rocas y de curtiembres (en especial porque no permiten la sostenibilidad del recurso 
Revista Tecné, Episteme y Didaxis: TED. Año 2014, Número Extraordinario. ISSN Impreso: 0121-3814, ISSN web: 2323-0126

Memorias, Sexto Congreso Internacional sobre Formación de Profesores de Ciencias. 08 al 10 de octubre de 2014, Bogotá

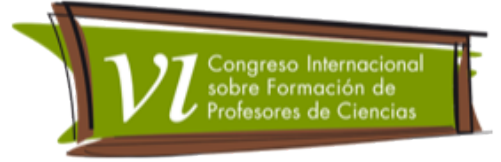

hídrico ya que no cumplen la normatividad existente), por la falta de servicios públicos, la poca funcionalidad de proyectos que buscan el cambio de cultura ambiental en los ciudadanos para disminuir el impacto de la contaminación atmosférica, la contaminación por ruido, la producción, inadecuado manejo y disposición final de residuos sólidos, la manipulación, riesgos de productos agroquímicos y las ventajas de utilizar la agricultura orgánica, . En los trabajos revisados se evidencia la necesidad de prevenir desastres y formar una cultura ambiental responsable en el manejo de estos, planteando de estrategias de formación y capacitación orientadas a aportar a los líderes comunitarios los conocimientos y a generar las actitudes, valores y comportamientos que les permita emprender acciones pro-ambientales.

\section{Conclusiones}

En el aula de clase se tiende a trabajar temas sobre la problemática ambiental detectada en el colegio o en la localidad más que con las inquietudes e intereses de los niños, en esta revisión se presentan trabajos de investigación etnográfica en donde se tiene en cuenta el sentir, la experiencia, las actitudes, las creencias, el pensamiento y las reflexiones de los sujetos pero no sus inquietudes e intereses. Se tratan temas de índole comunitario en los cuales se sale del aula para tratar de dar solución a situaciones que afectan a comunidades enteras por lo que es necesario hacer estudios históricos, demográficos, cartográficos, culturales, socioeconómicos y ambientales para abordarlos. Se evidencia como el docente puede detectar diferentes actores de la comunidad con los cuales puede trabajar para darle manejo al problema en cuestión como son los estudiantes, los maestros, los padres de familia, la familia, los líderes comunitarios, personas de sectores económicos, habitantes de zonas rurales y urbanas, usuarios de conjuntos residenciales, vendedores de fruta, entre otros.

La concientización y formación en valores juega un papel importante en la mayoría de los trabajos presentados ya que se cree que una conciencia ambiental logra cambiar las actitudes de daño ecológico y que con la planeación de una serie de actividades como talleres, caminatas, campañas, conformación de grupos ecológicos, conferencias, prácticas como cultivos, huertas, biocompóst, artes plásticas, entre otros con diferentes sujetos y en diferentes espacios se logrará una mejor calidad de vida y una cultura ambiental responsable. (Gómez H, 2000). Se evidencian propuestas pedagógicas como las 
Revista Tecné, Episteme y Didaxis: TED. Año 2014, Número Extraordinario. ISSN Impreso: 0121-3814, ISSN web: 2323-0126

Memorias, Sexto Congreso Internacional sobre Formación de Profesores de Ciencias. 08 al 10 de octubre de 2014, Bogotá

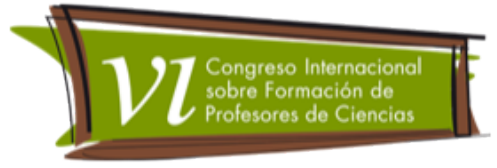

basadas en la lúdica y la educación artística y el diseño y manejo de software educativos. entre otros. Como lo afirma Cortés, Villalobos y Morales " La educación ambiental se asume en el aula con diversidad de posibilidades en donde la investigación, la curiosidad y la capacidad buscan ser privilegiadas" (Cortés, 2001). En estos procesos de sensibilización juega un papel muy importante la manipulación, la observación y la experimentación de la realidad ya que los niños aprenden más fácilmente " lo que viven" y si esto se combina con la lúdica que es la mejor forma de aprendizaje, el niño aprende y se siente feliz (Morales). Es así como El colegio puede ser un espacio de reflexión orientado hacia la formación de líderes para fomentar en la comunidad valores de solidaridad, respeto por la diferencia, búsqueda de conciencia y autonomía que preparen al individuo para la cogestión buscando siempre mejorar la calidad de vida. (Garcia J, 2000)

En cuanto a los residuos sólidos se evidencia el manejo en temas como: investigación en la dimensión ambiental, legislación ambiental, políticas para la gestión integral de residuos sólidos, políticas encaminadas a la clasificación, recolección y manejo de los residuos sólido y diseño de estrategias metodológicas educativas ambientales, como lo menciona Cobos, una conciencia de la producción de basura, su inadecuado manejo y disposición final, es y ha sido a través del tiempo uno de los principales factores del deterioro ambiental lo cual requiere de un cambio en la normatividad sobre el manejo de basuras, de una legislación que conduzca a la prestación de un servicio de aseo y de recolección selectiva de residuos, permitiendo una gestión de manejo integral de residuos en la fuente (Cobos M, 1999)

Las ATAs son una alternativa didáctica que permiten incorporar los intereses e inquietudes de los estudiantes para que no dependa del libro, del maestro, ya que lo que busca es que en la escuela, en vez de estudiarse "la flor que está en el libro", se estudie la flor del jardín, la flor del niño. Se trata de aventurarse a contradecir al texto y al manual o programa (Segura Dino \& Molina Adela, 2000, pág. 92), En nuestro caso se trata de que ellos se cuestionen y traten de resolver sus propios cuestionamientos específicamente en el manejo adecuado de los residuos sólidos producidos por ellos mismos.

\section{Referencias bibliográficas}

Bogotá, C. d. (2012). Colectivo Ciudadano Bogotá basura cero. Obtenido de http://www.bogotabasuracero.com/plan-desarrollo 
Revista Tecné, Episteme y Didaxis: TED. Año 2014, Número Extraordinario. ISSN Impreso: 0121-3814, ISSN web: 2323-0126

Memorias, Sexto Congreso Internacional sobre Formación de Profesores de Ciencias. 08 al 10 de octubre de 2014, Bogotá

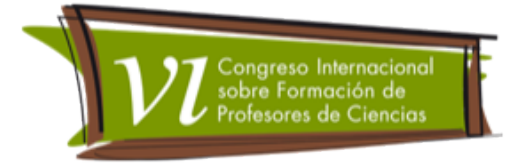

Cobos M, .. A. (1999). Por la implementación de un sistema de manejo de residuos domiciliarios en la cuenca del rio Tunjuelito. Bogotá: Universidad Distrital FJC.

Cortés, M. M. (2001). Proyecto para iniciar un cambio en la cultura ambiental en los niños de tercero de primaria en el Centro Educativo Distrital Venecia, Jornada mañana. Bogotá: Universidad Distrital FJC.

Garcia J, R. J. (2000). Evaluación de la funcionalidad de los proyectos ambientales escolares en tres colegios de Girardot con diferentes modalidades. Bogotá: Universidad Distrital FJC.

García, E. (2004). Educación Ambiental, Constructivismo y Complejidad. Sevilla.: Diada.

Gómez H, P. J. (2000). Propuesta pedagógica sobre el manejo adecuado de los residuos sólidos en la Unidad básica Agropecuaria y ambiental Las Auroras Localidad 20 ( Sumapaz). Bogotá: Universidad Distrital FJC.

Macias, I. (2008). Cliford Geertz y su visión sobre la cultura. México: Universidad de Guadalajara.

Molina, A. (2007). Analogía, pensamiento científico infantil y revalorzación de las teleologías y el antropomorfismo. Tecne Episteme Didaxis Número extraordinario, pág. 88-107.

Molina, A. (10-14 de Noviembre de 2013). Mapeamento Informacional Bibliográfico de Enfoque y Campos Temáticos de la diversidad cultural: el caso de las revistas CSSE, Sci EDu.And Sci. Academia.edu , 4.

Molina, A. (2005). Resumenes Analíticos Educativos RAE's, Universidad Distrital Francisco José de Caldas. Bogotá, Colombia: Fondo de Publicaciones Universidad Distrital F.J.C. Tomo I.

Morales, P. (s.f.). La importancia de que nuestros alumnos y alumnas reciclen. Obtenido de eduinnova.es: http://www.eduinnova.es/dic08/Que\%20recorrido\%20sigue\%20la\%20basura. pdf

Rivarosa, a. .. (2006). la resolución de problemas ambientales en la escuela y en la formción inicial de maestros. Revista Iberoamericana de de educación . 
Revista Tecné, Episteme y Didaxis: TED. Año 2014, Número Extraordinario. ISSN Impreso: 0121-3814, ISSN web: 2323-0126

Memorias, Sexto Congreso Internacional sobre Formación de Profesores de Ciencias. 08 al 10 de octubre de 2014, Bogotá

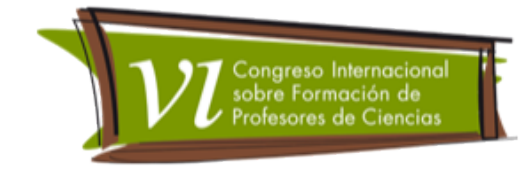

Robayo, J. (1999). Cómo implementar la educación ambiental en los estudiantes del grado sexto de la institución Centro Educativo INEM Santiago PerezTunal - Bogotá. Bogotá: tesis de Grado.

Sanmarti, N. (2010). Enseñar y aprender ciencias: Algunas reflexiones. http://www.guiasensenanzasmedias.es/verpdf.asp? area=natura \& $\operatorname{archivo}=\mathrm{G}$ R104.pdf.

Schön, D. (1992). La formación de profesionales reflexivos. Hacia un nuevo diseño de la enseñanza y el aprendizaje en las profesiones. En D. Schön, La formación de profesionales reflexivos. Hacia un nuevo diseño de la enseñanza y el aprendizaje en las profesiones. (págs. 54-265). Barcelona: Paidos.

Segura, D. M. (2000). Vivencias de conocimiento y cambio cultural. Bogotá: Escuela pedagógica esperimental.

Segura, D. y. (2000). Vivncias de conocimiento y cambio cultural. Bogotá: Escuela pedagógica experimental.

Villalobos, F. (2001). Propuesta pedagógica-metodológica de educación ambiental como dimensión del desarrollo humano en el colegio Ateneo Integral. Bogotá: Universidad Distrital FJC. 